\title{
Efeito do processamento em misturas de alumina/ligantes orgânicos usadas na moldagem por injeção em baixa pressão
}

\section{(Effect of processing variables in alumina/organic binders mixtures used in low-pressure injection molding)}

\author{
P. A. Ourique, A. Susin Neto, S. G. Echeverrigaray, R. C. D. Cruz, J. E. Zorzi \\ Instituto de Materiais Cerâmicos, Universidade de Caxias do Sul, 95765-000, Bom Princípio, RS, Brasil \\ rcdcruz@ucs.br
}

\begin{abstract}
Resumo
A moldagem por injeção em baixa pressão (MIBP) é uma técnica que já vem sendo empregada na produção de peças cerâmicas com formas e geometrias complexas. A homogeneidade da mistura de ligantes orgânicos e pós cerâmicos é um fator determinante que deve ser controlado para minimizar a formação de imperfeições no processamento de feedstocks para MIBP. Defeitos típicos de processamento por MIBP, como bolhas de ar e aglomerados, geram gradientes de densidade nas misturas que, após conformação, possuem poucas possibilidades de remoção. Essas imperfeições comprometem o desempenho dos produtos obtidos por essa técnica. Este trabalho está focado na avaliação dessas heterogeneidades e como elas podem ser correlacionadas com a variação da densidade aparente e com o comportamento reológico dessas misturas. Para tanto, aluminas submicrométricas, como recebida e desaglomerada, foram adicionadas a uma mistura fundida de ligantes a base de parafinas, ceras e aditivos e processada em dois tipos diferentes de misturadores, com e sem o auxílio de vácuo. Foi observada a presença de aglomerados existentes na alumina como recebida, possivelmente gerados durante a etapa de calcinação. Também foi observado que o tipo de misturador e a aplicação ou não de vácuo durante a etapa final do processamento têm grande influência no tempo de mistura necessário para reduzir a viscosidade do feedstock para a injeção.
\end{abstract}

Palavras-chave: moldagem por injeção em baixa pressão, alumina, ligantes, densidade a verde, reologia, processamento.

\begin{abstract}
The low-pressure injection molding (LPIM) is a technique already being used in the production of ceramic parts with complex shapes and geometries. The homogeneity of the mixture of organic binder and ceramic powder is a determining factor which must be controlled to minimize defects formation while feedstock processing to LPIM. Typical defects of LPIM processing, such as air bubbles and agglomerates, generate density gradients in the mixtures, which, after shaping, have little possibility of removal. These imperfections compromise the performance of the products obtained by this technique. This work is focused on the evaluation of these inhomogeneities and how they can be correlated with density variation and the rheological behavior of these mixtures. Therefore, submicrometer aluminas, as received and deagglomerated, were added to a molten mixture of paraffin based binders, waxes and additives and processed in two different mixers, with and without vacuum. The presence of alumina agglomerates was observed in the powder as received, possibly generated during the calcination step. It was also observed that the type of mixer and vacuum application or not during the final processing step, has a major influence on the mixing time required to reduce the viscosity of the feedstock for injection.
\end{abstract}

Keywords: low-pressure injection molding, alumina, binder, green density, rheology, processing.

\section{INTRODUÇÃO}

Os processos de moldagem de pós cerâmicos por injeção envolvem a mistura de pós inorgânicos com ligantes orgânicos e agentes de superfície. $\mathrm{O}$ grau e a forma de interação físico-química entre os componentes da mistura influenciam o comportamento reológico da massa, e conseqüentemente o empacotamento das partículas, formação de aglomerados, processabilidade, extração dos ligantes, precisão dimensional e também a origem dos defeitos nas peças finais sinterizadas. A metodologia de mistura para formar uma massa homogênea para injeção, bem como a natureza dos ligantes e agentes de superfície, varia dependendo do pó de partida e do processo de moldagem a serem empregados. Na moldagem por injeção em baixa pressão (MIBP), quando comparada com a moldagem em alta pressão, a temperatura e a pressão de injeção são consideravelmente menores (até $\sim 150{ }^{\circ} \mathrm{C}$ e $\sim 0,6 \mathrm{MPa}$ ), os ligantes são usualmente ceras naturais e sintéticas, os moldes podem ser fabricados com materiais alternativos, e.g. latão, resinas poliméricas e recobertos com filme antiaderente e a mistura dos componentes é produzida na própria injetora (Fig. 1). Estes fatores tornam o processo economicamente atrativo para a fabricação de componentes de geometria complexa [1-4]. O tempo de mistura para o feedstock preparado para MBIP geralmente compreende várias horas. As injetoras de baixa pressão possuem o inconveniente de ter uma única câmara compartilhada para mistura e 
armazenagem do feedstock de injeção, o que impossibilita a moldagem de componentes simultaneamente a realização de misturas. A câmara de mistura das injetoras de baixa pressão é composta basicamente por um reservatório aquecido com misturador integrado e conectada a uma bomba de vácuo [5]. Essa construção integrada das injetoras de baixa pressão abre a oportunidade de se avaliar o emprego de outros tipos de misturador externo a injetora para preparar uma mistura à parte e acelerar o processo de fabricação.

Este trabalho teve como objetivo avaliar o efeito do uso de um misturador planetário externo na redução do tempo de mistura no interior da injetora. Foi avaliado como parâmetro de controle do processamento a densidade à verde e o comportamento reológico do feedstock. Outros fatores importantes para minimizar o tempo de mistura, como a

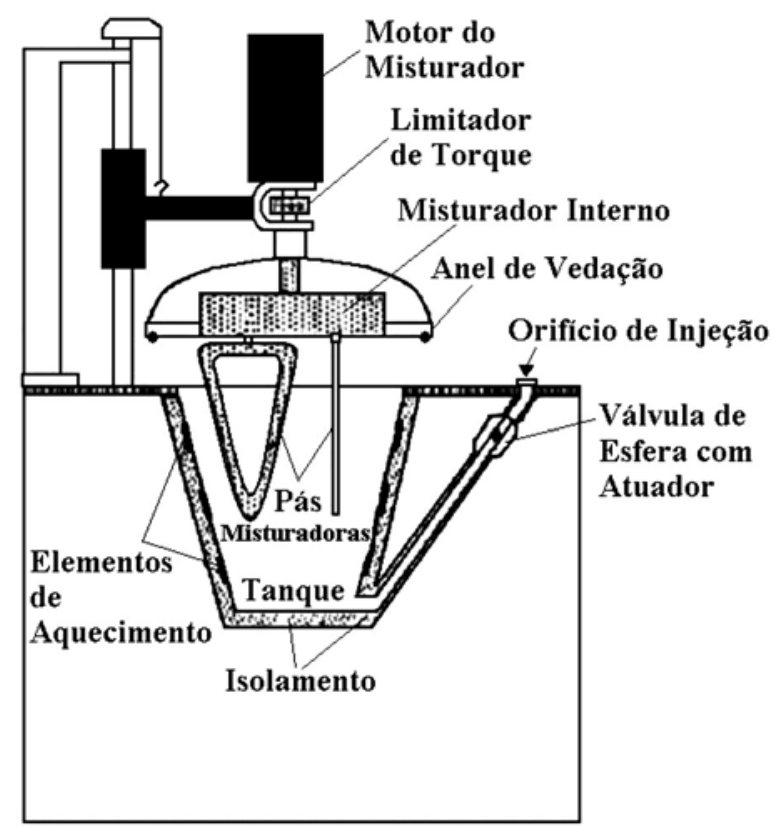

Figura 1: Representação esquemática de injetora de baixa pressão [2].

[Figure 1: Schematic representation of low-pressure injection molding machine [2].]

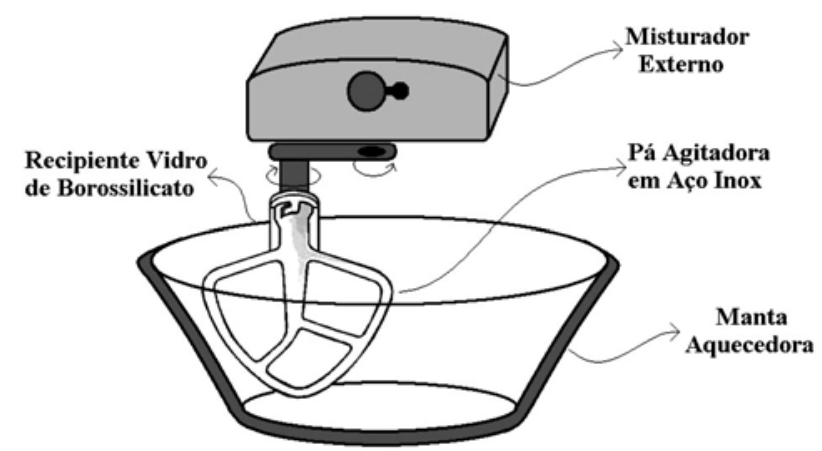

Figura 2: Esquema do misturador externo, com recipiente de vidro de borossilicato, envolto por manta aquecedora.

[Figure 2: Schematic of planetary mixer with borosilicate glass container, wrapped in a heating mantle.] distribuição de tamanho das partículas do pó de partida e vácuo, também foram avaliados. Os resultados indicam quais as melhores condições de processamento que levam a uma mistura com baixa viscosidade e alta fração de sólidos. Foram utilizados dois equipamentos: uma injetora de baixa pressão, a qual possui um misturador interno com duas pás (Fig. 1) e um misturador de uso comercial, com uma única pá (Fig. 2), ambos com movimento planetário.

\section{MATERIAIS E MÉTODOS}

O pó cerâmico utilizado foi $\alpha$-alumina calcinada A-1000SG (Almatis Inc., Leetsdale, PA, EUA). A composição é $99,8 \% \mathrm{Al}_{2} \mathrm{O}_{3}, 0,07 \% \mathrm{~N}_{2} \mathrm{O}, 0,03 \% \mathrm{SiO}_{2}, 0,02 \%$ $\mathrm{Fe}_{2} \mathrm{O}_{3}, 0,02 \% \mathrm{CaO}$ e $0,04 \% \mathrm{MgO}$ (peso\%), área superficial do pó de $8,4 \mathrm{~m}^{2} / \mathrm{g}$ e tamanho médio de partícula $0,4 \mu \mathrm{m}$ (dados do fabricante). A alumina como recebida (ACR) foi seca em estufa a $120^{\circ} \mathrm{C} / 3 \mathrm{~h}$ previamente à etapa de mistura. $\mathrm{O}$ veículo orgânico empregado foi composto por cera de parafina, cera de carnaúba, cera de polietileno, ácido oleico e esteárico [6].

A mistura utilizada foi composta por $86 \%$ p de alumina e $14 \%$ p de veículo orgânico ( 45vol.\%.) [6]. Inicialmente os componentes orgânicos foram fundidos dentro do misturador previamente aquecido a $90{ }^{\circ} \mathrm{C}$. O pó foi adicionado em alíquotas sob agitação constante. Os componentes do feedstock foram misturados em intervalos de $10 \mathrm{a} 70 \mathrm{~h}$.

Foram utilizados um misturador externo KitchenAid K45ss com uma pá, esquematizado na Fig. 2, com recipiente de vidro de borossilicato envolto por manta de lã cerâmica com aquecimento e uma injetora de baixa pressão, Peltsman MIGL-33 (semiautomática) (Fig. 1), com misturador de duas pás dentro do tanque e bomba de vácuo acoplada. Ambos misturadores possuem movimento planetário. As misturas ficaram sob agitação e vácuo por $1 \mathrm{~h}$ antes da injeção. $\mathrm{O}$ molde foi posicionado na abertura do tubo de saída (também aquecido) e a moldagem ocorreu com a injeção de ar comprimido sobre a mistura fundida.

As amostras foram injetadas em molde metálico em formato de disco de $22 \mathrm{~mm}$ de diâmetro e $3 \mathrm{~mm}$ de espessura, com pressão de $400 \mathrm{kPa}$ e tempo de injeção $12 \mathrm{~s}$. Antes de cada injeção o molde foi lubrificado com óleo de silicone. Concluídas as injeções o molde foi removido da injetora e refrigerado ao ar por $1 \mathrm{~min}$.

Para avaliar os efeitos do pó de partida no processamento do feedstock, experimentos semelhantes aos descritos acima foram conduzidos para a alumina desagregada (AD). A alumina foi desagregada em água em um moinho de esferas cilíndrico, com revestimento do moinho e esferas de alumina, com capacidade para $5 \mathrm{~L}$. O tempo de desagregação foi 24 h, a $32 \mathrm{rpm}$, com $80 \%$ do volume do moinho ocupado por esferas, pó e água destilada e deionizada. A seguir o pó foi seco em estufa e desaglomerado manualmente em almofariz. Após ser seco novamente a $120^{\circ} \mathrm{C} / 3 \mathrm{~h}$, o pó foi adicionado e redispersado no veículo orgânico fundido.

Amostras injetadas da ACR e da AD foram sinterizadas ao ar até $1600{ }^{\circ} \mathrm{C}$ (forno Lindberg/Blue M, Asheville, 
$\mathrm{NC}$, EUA) com taxa de aquecimento $1{ }^{\circ} \mathrm{C} / \mathrm{min}$ e patamar $2 \mathrm{~h}$. Para revelar e avaliar a microestrutura resultante, as amostras foram polidas com pastas diamantadas, atacadas termicamente a $1450{ }^{\circ} \mathrm{C} / 0,5 \mathrm{~h}$, recobertas com ouro e analisadas por microscopia eletrônica de varredura (microscópio Shimadzu SSX-550, Kyoto, Japão).

As densidades das amostras a verde e sinterizadas, processadas na injetora e no misturador planetário externo, foram medidas pelo método de Arquimedes [7] em uma balança com precisão de $0,1 \mathrm{mg}$. Imagens da superfície de fratura das amostras a verde foram obtidas em um microscópio óptico Zeiss Axioscope A1(Carl Zeiss GmbH, Göttingen, Alemanha).

Medidas da distribuição de tamanho de partícula (a úmido), da $\mathrm{ACR}$ e da $\mathrm{AD}$, foram obtidas pelo método de espalhamento de laser, em um analisador Horiba Partica LA-950V2 (Kyoto, Japão), sem auxílio de ultrassom durante a medição.

As propriedades reológicas das misturas foram analisadas em um reômetro oscilatório Physica MCR 101 (Anton Paar GmbH, Graz, Austria), usando o sistema de medida placa-placa PP 25-2. As medidas foram obtidas a 90 ${ }^{\circ} \mathrm{C}$, com tensão de cisalhamento constante $10 \mathrm{~Pa}$ e freqüência angular variada de 100 a $0,1 \mathrm{rad} / \mathrm{s}$. As medidas foram feitas dentro da região de viscoelasticidade linear.

\section{RESULTADOS E DISCUSSÃO}

Na Fig. 3 são apresentados os resultados das medidas de distribuição de tamanho de partícula da alumina antes e após a etapa de desagregação do pó. O pó, mesmo tendo sido previamente moído (ACR), apresenta uma distribuição bimodal com presença de aglomerados na faixa de 1-30 $\mu \mathrm{m}$, oriundos possivelmente da calcinação do pó, que não foram destruídos durante a moagem realizada pelo fornecedor. Essa distribuição é caracterizada por apresentar um $d_{10}$ $=0,19, \mathrm{~d}_{50}=0,38$ e $\mathrm{d}_{90}=7,84 \mu \mathrm{m}$. É bem conhecido que a distribuição multimodal de partículas é adequada para melhorar o empacotamento à verde de produtos cerâmicos [8], porém deletéria para maximizar a densificação do corpo verde após a etapa de sinterização, contribuindo para a redução da confiabilidade do desempenho mecânico do produto final [9]. Portanto a alteração da distribuição de tamanhos de bi- para monomodal é aconselhável e desejável para uniformizar o tamanho das partículas do pó de partida. A alteração da distribuição de tamanho da ACR foi possível após desaglomeração como mostra a Fig. 3. A ausência quase total dos aglomerados primários é evidente e a distribuição resultante se caracteriza por apresentar um $\mathrm{d}_{10}$ $=0,18, \mathrm{~d}_{50}=0,29$ e $_{90}=1,40 \mu \mathrm{m}$. Nas condições adotadas o pó não apresentou alteração na fração $\mathrm{d}_{10}$, indicando que durante esta operação ocorreu apenas desaglomeração e não moagem.

Na Tabela I e Fig. 4 são apresentadas as densidades das amostras a verde e sinterizadas, injetadas em diferentes condições de processamento. A fim de ressaltar o efeito da desaglomeração nas características do feedstock e do produto

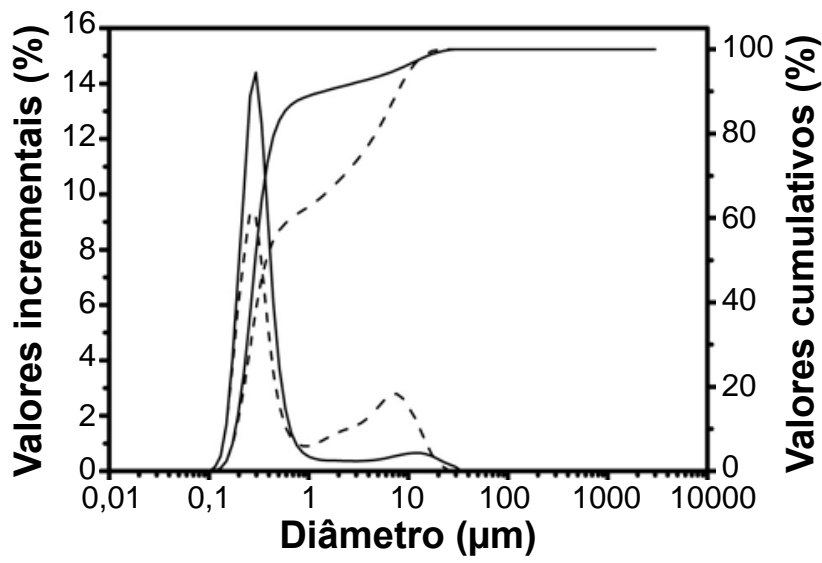

Figura 3: Distribuição mássica do tamanho das partículas da ACR (--) e da AD (-) .

[Figure 3: Mass-size distribution of alumina particles as received (--) and deagglomerated (-).]

final, foram selecionados os resultados para amostras após $40 \mathrm{~h}$ de mistura e sob vácuo. Os valores de densidade variaram de 2,668 a $2,646 \mathrm{~g} / \mathrm{cm}^{3}$ e 3,863 a $3,900 \mathrm{~g} / \mathrm{cm}^{3}$ para as amostras a verde e sinterizadas, respectivamente. $\mathrm{O}$ maior valor de densidade a verde obtido foi para a amostra injetada com a ACR, resultado que confirma o efeito da distribuição bimodal no empacotamento, provocado pela presença de aglomerados. Como esperado, este arranjo volumétrico da ACR não conduz a uma densificação elevada da estrutura sinterizada, Fig. 5, tanto que, como indicado na Fig. 4, o maior valor de densidade para as amostras sinterizadas resultou das peças injetadas com menor densidade a verde. Por estes motivos, a desaglomeração do pó de partida é recomendável quando se deseja obter peças cerâmicas com estrutura final homogênea. Além disso, a etapa de desagregação permite que ofeedstock seja produzido em tempos curtos de mistura em comparação àqueles obtidos para o pó original.

A Fig. 6 apresenta os resultados das medidas de densidade à verde versus tempo de mistura no misturador externo comparando a $\mathrm{AD}$, com e sem aplicação de vácuo, com a ACR, sem vácuo. Independente do tempo de mistura, a desaglomeração do pó de partida resultou em altos valores de densidade à verde e em baixa variação da densidade do corpo injetado, comportamento que é concordante com uma distribuição de tamanho monomodal, Fig. 6 , curvas $(\Delta)$ e $(\bullet)$. Este comportamento contrasta com o apresentado pela ACR, a qual apresentou variação da densidade em toda a

Tabela I - Medidas de densidade de amostras preparadas na injetora após $40 \mathrm{~h}$ de mistura com vácuo.

[Table I - Density measurements of samples prepared in the LPIM machine after 40 h of mixture with vacuum.]

\begin{tabular}{ccc}
\hline Pó de partida & $\begin{array}{c}\text { Densidade à } \\
\text { verde }\left(\mathrm{g} / \mathrm{cm}^{3}\right)\end{array}$ & $\begin{array}{c}\text { Densidade } \\
\text { sinterizada a } \\
1600{ }^{\circ} \mathrm{C}\left(\mathrm{g} / \mathrm{cm}^{3}\right)\end{array}$ \\
\hline $\mathrm{ACR}$ & $2,668 \pm 0,001$ & $3,863 \pm 0,002$ \\
$\mathrm{AD}$ & $2,646 \pm 0,002$ & $3,900 \pm 0,001$ \\
\hline
\end{tabular}




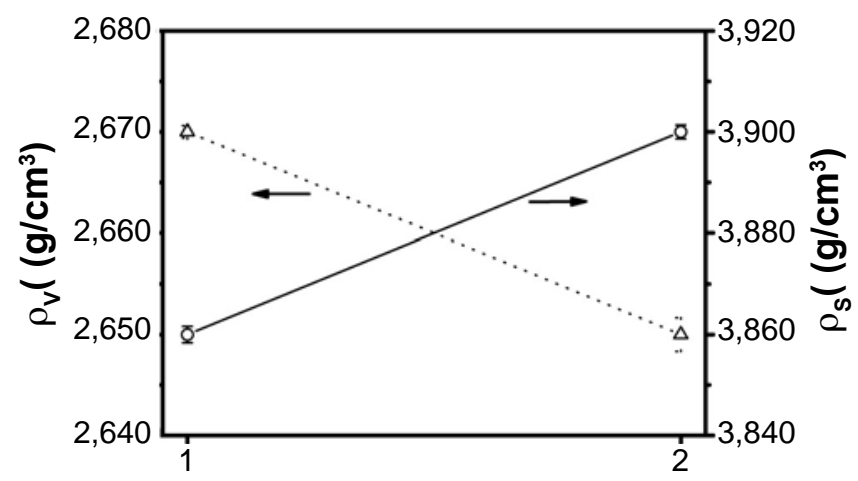

Condição de processamento

Figura 4: Variação das densidades a verde $\left(\rho_{v}\right)$ e sinterizadas $\left(\rho_{s}\right)$, em $\mathrm{g} / \mathrm{cm}^{3}$, das amostras misturadas por $40 \mathrm{~h}$ na injetora (com vácuo), em função das condições de processamento: (1) ACR e (2) AD.

[Figure 4: Variation of densities of green $\left(\rho_{v}\right)$ and sintered samples $(\rho)$, in $\mathrm{g} / \mathrm{cm}^{3}$, mixed for $40 \mathrm{~h}$ in LPIM machine (with vacuum), depending on the processing conditions: (1) as received alumina and (2) deagglomerated alumina.]
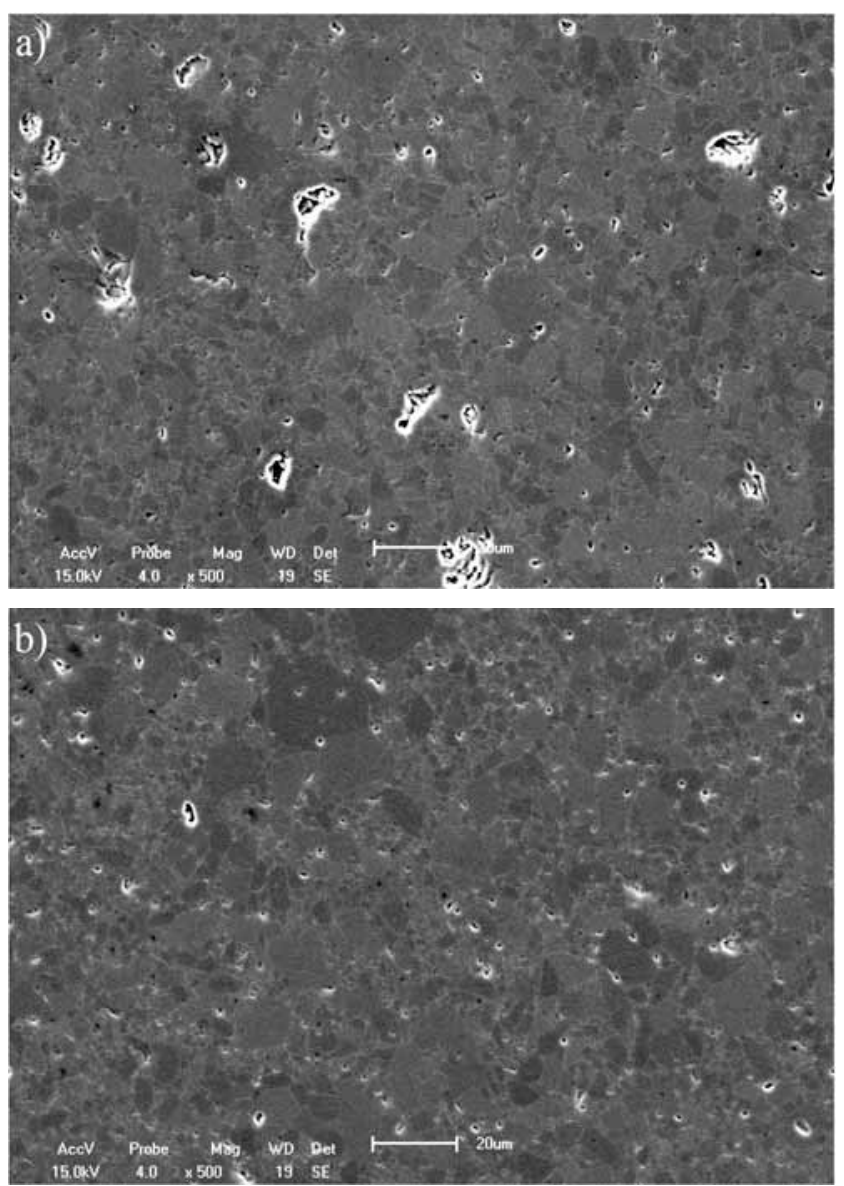

Figura 5: Microestrutura das amostras sinterizadas a $1600{ }^{\circ} \mathrm{C} / 2 \mathrm{~h}$, polidas e atacadas termicamente a $1450{ }^{\circ} \mathrm{C} / 0,5 \mathrm{~h}$. a) ACR e b) AD. [Figure 5: Microstructure of samples sintered at $1600{ }^{\circ} \mathrm{C} / 2 \mathrm{~h}$, polished and thermally etched at $1450{ }^{\circ} \mathrm{C} / 0.5 \mathrm{~h}$. a) as received alumina and b) deagglomerated alumina.]

faixa de tempo de mistura avaliado, Fig. 6, curva (o). Essa dependência do tempo de mistura é justificada já que o pó apresenta uma fração de aglomerados sujeitos a esforços

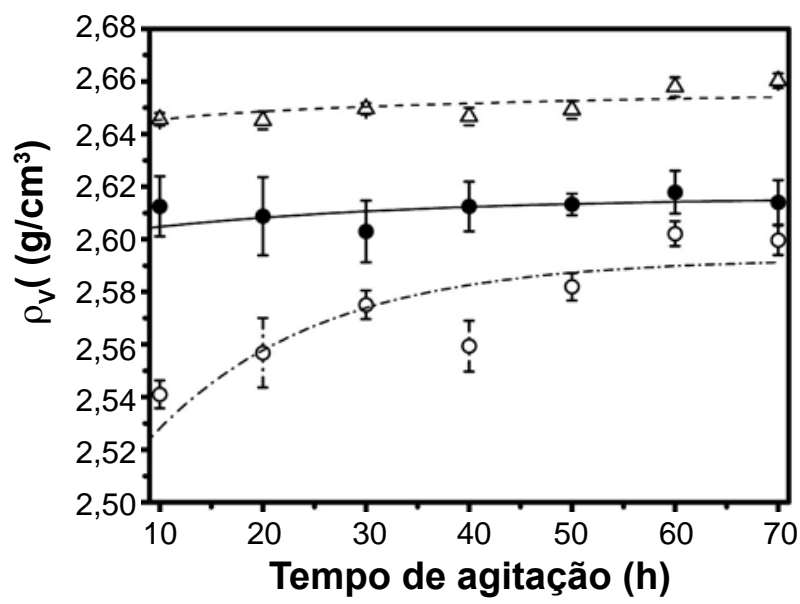

Figura 6: Variação da densidade à verde versus tempo de mistura no misturador externo: $(\Delta) \mathrm{AD}$ com vácuo, $(\bullet) \mathrm{AD}$ sem vácuo e $(0)$ ACR sem vácuo.

[Figure 6: Green density variation versus mixing time in the external mixer: alumina $(\Delta)$ deagglomerated with vacuum, (•) deagglomerated without vacuum, and ( $\mathrm{O})$ as received without vacuum.]

de cisalhamento impostos pelo misturador, os quais são progressivamente destruídos durante a mistura. Assim sendo, a estrutura resultante é diretamente dependente do tempo de residência do feedstock no misturador. Além disso, quanto maior o tempo de mistura, maior a probabilidade dos agentes dispersantes adsorverem na superfície das partículas. Após 70 h de mistura, a interação partícula-ácidos graxos se torna efetiva para a ACR, o que resulta em um material com densidade muito próxima a densidade da $\mathrm{AD}$ nas mesmas condições de processamento.

Para as amostras do pó desaglomerado, os maiores valores de densidade à verde foram obtidos com aplicação de vácuo, Fig. 6, curva $(\Delta)$. Durante a adição do pó cerâmico à fase orgânica fundida é inviável evitar a formação de aglomerados fracos, fato que acarreta o aprisionamento de ar entre as partículas de pó. Dada a elevada viscosidade da fase líquida formada pelo veículo orgânico na temperatura de mistura, o ar presente na superfície das partículas e/ou aglomerados e que é parcialmente liberado, tende a formar bolhas. Por sua vez, as bolhas que ficam aprisionadas na fase líquida, coalescem durante a mistura e geram macrodefeitos quando do resfriamento da peça após injeção. A análise microscópica da superfície de fratura de amostra à verde da ACR e sem vácuo (Fig. 7) permite evidenciar a existência de bolhas, geradas durante o processo de mistura, resultando numa massa de injeção heterogênea e trifásica. Esta estrutura obtida sem nenhum tipo de tratamento especial possui a mais baixa densidade medida nesta série experimental, $2,610 \mathrm{~g} / \mathrm{cm}^{3}$.

Em trabalho recente, foi mostrado que a porosidade residual é extremamente crítica para o desempenho mecânico de molas de alumina injetadas em baixa pressão. Mesmo pequenas variações de densidade após a sinterização reduzem significativamente a carga que as molas suportam antes de romper [10].

A fim de comprovar que se pode usufruir de um sistema 


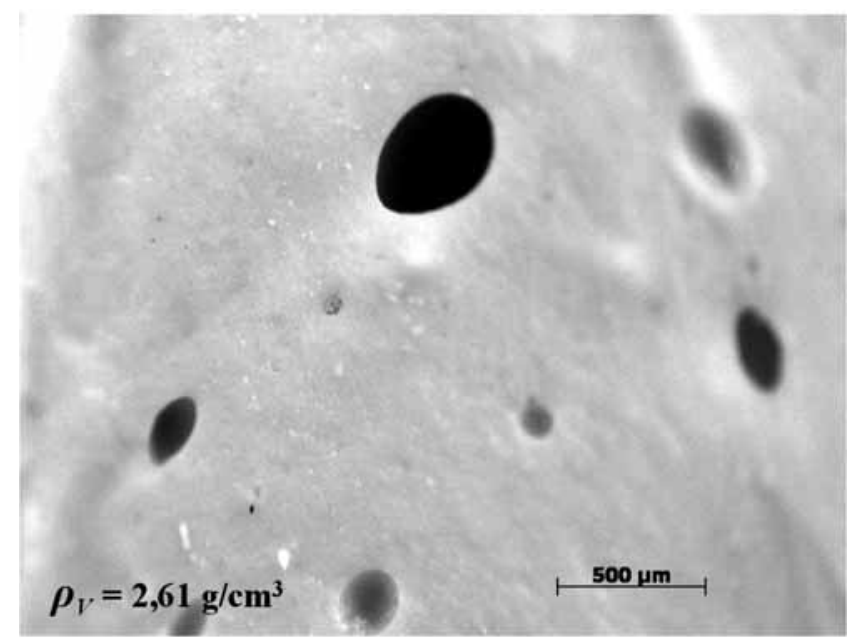

Figura 7: Imagem obtida por microscopia óptica (50x) da superfície de fratura à verde da ACR injetada sem vácuo, após $60 \mathrm{~h}$ de mistura. [Figure 7: Image obtained by optical microscopy (50x) of green sample fractured surface of as received alumina injected without vacuum, after 60 h of mixing.]

independente de preparação e mistura de feedstock e utilizar a injetora como tal, uma amostra da $\mathrm{AD}$ e misturada por 70 $\mathrm{h}$ no misturador externo foi colocada na injetora, submetida a vácuo por $1 \mathrm{~h} \mathrm{e}$ injetada. A densidade das amostras sinterizadas produzidas por esse método combinado de preparação foi de $3,888 \pm 0,001 \mathrm{~g} / \mathrm{cm}^{3}$. Estes valores são semelhantes aos obtidos para os feedstocks processados somente na injetora. Desta forma, concluiu-se que o produto final resultante de misturas realizadas em misturador externo pode ter a mesma densificação de amostras preparadas diretamente no misturador da injetora.

A robustez dos processos de conformação por injeção sob pressão de pós cerâmicos está diretamente associada ao desempenho mecânico do feedstock, desde sua mistura e homogeneização, passando pela etapa de enchimento da cavidade da matriz de conformação até a desmoldagem do corpo verde que originará o produto final desejado. Cada uma destas etapas é diretamente dependente da resistência ao escoamento oferecida pelas misturas, bem como das demais variáveis reológicas que determinam sua estabilidade ao longo dos tempos característicos das etapas de pré-injeção, injeção e pós-injeção. Assim sendo, ensaios reológicos dinâmicos foram conduzidos para avaliar o desempenho global das misturas para moldagem por injeção em baixa pressão (MIBP). A Fig. 8 mostra os resultados da variação dos módulos de armazenamento (G') e de perda (G") em função da freqüência angular para o misturador externo após 20 e 60 h de agitação com a ACR e sem vácuo. É evidente a predominância do caráter elástico do feedstock uma vez que G' é maior que G" para toda a faixa de freqüências analisadas. Esta resposta revela o comportamento típico de sólido viscoelástico das misturas para MIBP [11].

É amplamente propagado na literatura que misturas eficientes entre o pó e a fase contínua, para uma mesma concentração de sólidos, resultam em estruturas a verde, mais homogêneas e são a causa para a redução da viscosidade

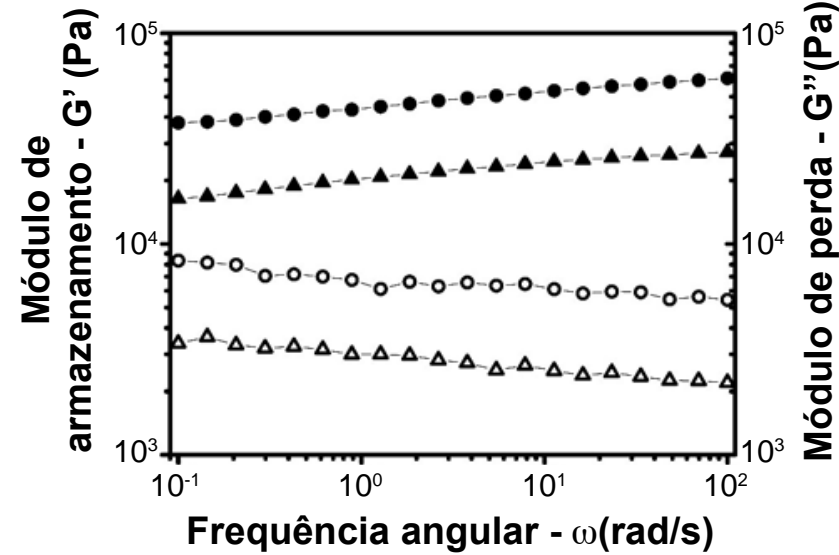

Figura 8: Módulos de armazenamento (G’) (símbolos cheios) e de perda (G”) (símbolos vazados) versus freqüência angular, para a ACR no misturador externo, sem vácuo: ( $\mathrm{O}$ ) após $20 \mathrm{~h}$ de mistura e $(\Delta)$ após $60 \mathrm{~h}$ de mistura.

[Figure 8: Storage modulus ( $\left.G^{\prime}\right)$ (filled symbols) and loss modulus $(G$ ") (open symbols) versus angular frequency, for as received alumina using external mixer, without vacuum: (०) after $20 \mathrm{~h}$ of mixing and $(\Delta)$ after 60 h of mixing.]

dessas misturas [12], ou seja, melhor empacotamento requer menor quantidade da fase contínua para fluir ofeedstock. De maneira concordante ao que foi observado para a densidade a verde, o aumento do tempo de agitação do feedstock possibilita melhor distribuição da fase polimérica entre as partículas rígidas de alumina, o que resulta em uma mistura mais homogênea e menos elástica, i.e., $\mathrm{G}_{60 \mathrm{~h}}$ é menor que $\mathrm{G}_{20 \mathrm{~h}}$. Nestas misturas bifásicas a função dos ácidos graxos, oleico e esteárico, como agentes modificadores de superfície, é possibilitar a compatibilização entre as superfícies polares das partículas de alumina e a matriz apolar polimérica, a fim de reduzir a interação das partículas cerâmicas entre si e com a matriz orgânica. Com o aumento do tempo de residência da mistura no misturador externo, as possibilidades de contato partículas-ácidos graxos também aumentam, o que facilita a adsorção destes aditivos à superfície da fase sólida. Com a conseqüente diminuição de aglomerados resultantes da redução do contato partícula-partícula causada pela ação dos ácidos graxos durante a mistura, é possível obter-se valores reduzidos de viscosidade, $\eta_{60 \mathrm{~h}}^{*}$ é menor que $\eta^{*}{ }_{20 \mathrm{~h}}$, Fig. 9. Este resultado reológico confirma a possibilidade de se produzir feedstocks com carga volumétrica de sólidos maior, uma vez que valores experimentais acima de $65 \%$ vol. são relatados [13]. Deve-se ter em mente que os valores da literatura foram obtidos para partículas com $\mathrm{d}_{50}=0,54-1,3 \mu \mathrm{m}$, tamanhos médios superiores ao utilizado neste trabalho, o que evidencia quão determinante é esta característica do pó para o processamento do feedstock.

De maneira geral, pode-se depreender destes experimentos que quanto mais intensivo for o tratamento, e.g. vácuo, no feedstock e maiores os tempos de agitação, menores serão as viscosidades das misturas e melhor será o empacotamento da fase sólida. Estas variáveis de processamento atingem um valor limite, o qual ocorrerá para 


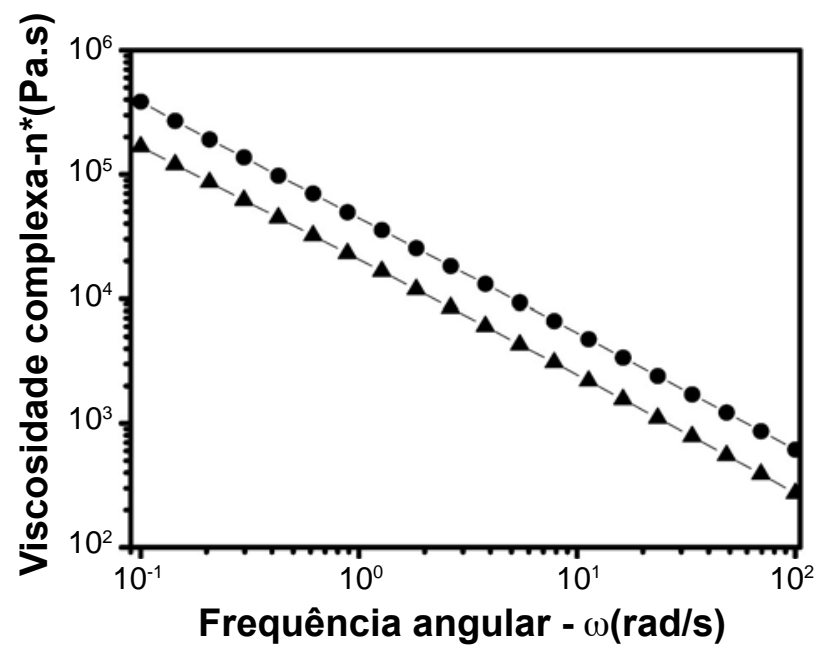

Figura 9: Viscosidade complexa versus freqüência angular para ACR sem vácuo no misturador externo (•) após $20 \mathrm{~h}$ de mistura e ( $\Delta$ ) após $60 \mathrm{~h}$ de mistura.

[Figure 9: Complex viscosity versus angular frequency for as received alumina, using the external mixer without vacuum (•) after 20 h of mixing and ( $\mathbf{\Delta})$ after 60 h of mixing.]

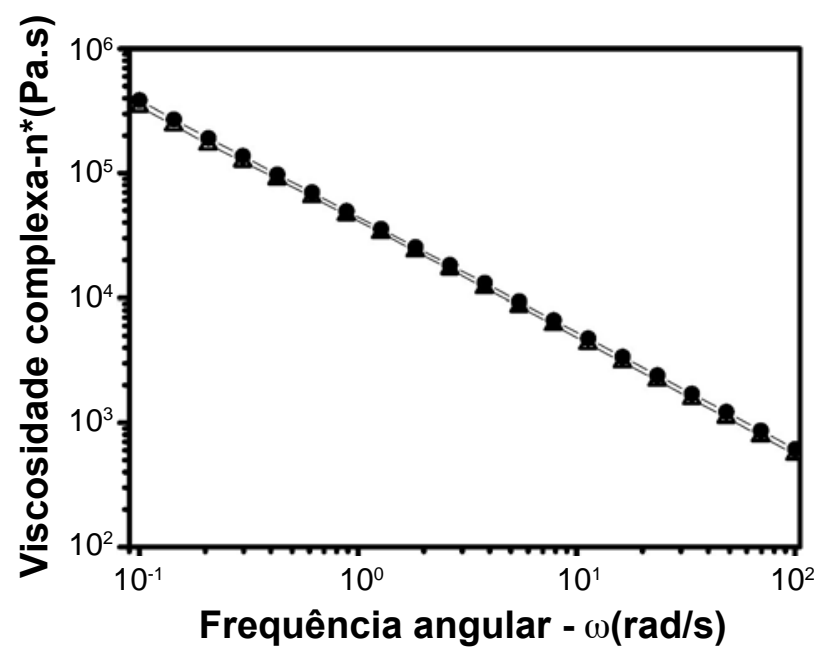

Figura 10: Viscosidade complexa versus freqüência angular para a ACR com $20 \mathrm{~h}$ de mistura sem vácuo $(\Delta)$ na injetora e $(\bullet)$ no misturador externo.

[Figure 10: Complex viscosity versus angular frequency for as received alumina after $20 \mathrm{~h}$ of mixing without vacuum $(\Delta)$ in the LPIM machine and $(\bullet)$ in the external mixer.]

tempos de misturas que são diretamente dependentes das condições de processamento adotadas e das características do pó de partida. Esses tempos podem ser relativamente longos, $>50 \mathrm{~h}$ para o feedstock processado no misturador externo com ACR, ou curtos, $\sim 20 \mathrm{~h}$ para o feedstock processado na injetora com vácuo com $\mathrm{AD}$.

Para demonstrar a viabilidade da substituição da injetora pelo misturador externo, amostras foram ensaiadas na condição menos favorável do ponto de vista de homogeneização, i.e., sem desaglomeração e sem vácuo por um tempo de mistura de somente 20 h. A Fig. 10 mostra as medidas de viscosidade complexa versus freqüência angular para ofeedstock preparado no misturador externo e na injetora na condição descrita. A mistura realizada no misturador externo exibe um comportamento reológico semelhante àquela preparada na injetora, com valor levemente maior, não comprometendo a etapa de injeção de peças, mesmo se for considerado que a taxa máxima de cisalhamento calculada para o misturador externo seja levemente menor que para a injetora, $6 \mathrm{~s}^{-1}$ e $23 \mathrm{~s}^{-1}$, respectivamente [14].

A obtenção de peças cerâmicas injetadas com resistência mecânica controlada, na fabricação de produtos acabados com tolerância dimensional estreita, é diretamente dependente das etapas pós-injeção do feedstock [15]. Para avaliar o grau desta dependência, testes variando parâmetros de injeção, como pressão e tempo, e pós injeção, como envelhecimento do corpo à verde e condições para remoção do veículo orgânico, estão sendo conduzidos no momento e serão publicados futuramente.

\section{CONCLUSÕES}

As misturas com a AD apresentaram densidades após sinterização superiores às amostras que foram obtidas com a ACR, justificando assim a desaglomeração do pó antes da mistura com os componentes orgânicos. Também foi observado que a ação do vácuo durante a mistura tem grande influência na viscosidade do feedstock. De fato, a presença de bolhas de ar é fator predominante para o controle da viscosidade e densidade final da mistura para injeção.

A mistura realizada no misturador externo exibiu um comportamento reológico similar ao da mistura preparada no tanque da própria injetora. Deste modo, pode-se usar um misturador planetário tipo comercial, com pá simples, e realizar a mistura dos ligantes com o pó cerâmico desde que seja aplicado vácuo por pelo menos $1 \mathrm{~h}$ antes da injeção do feedstock, sempre em agitação. Condições de processamento que levam a uma mistura com baixa viscosidade e alta fração de sólidos são indicadas.

\section{AGRADECIMENTOS}

Os autores agradecem o apoio financeiro de FAPERGS, CAPES, FINEP e CNPq para a realização deste trabalho. Ao LPA da UFRGS e ao LASAN e LPol da UCS pelo empréstimo de equipamentos. Ao Prof. Dr. Cláudio A. Perottoni pelas discussões e ao bolsista de iniciação científica, Matias S. Lunkes, pelo auxílio nas medidas de densidade e microscopia.

\section{REFERÊNCIAS}

[1] J. A. Mangels, Am. Ceram. Soc. Bull. 73, 5 (1994) 37. [2] J. E. Zorzi, C. A. Perottoni, J. A. H. da Jornada, Cerâmica 50, 315 (2004) 202-208.

[3] W. Bauer, M. Gómez, V. Valcárcel, C. Cerecedo, F. Guitián, M. Peltsman, J. E. Zorzi, Ceram. Ind. 156, 5 (2006) 22-26.

[4] I. Krindges, R. Andreola, J. E. Zorzi, C. A. Perottoni, Int. 
J. Appl. Ceram. Tech. 5, 3 (2008) 243-248.

[5] I. Peltsman, M. Peltsman, Interceram. 4 (1984) 56.

[6] J. E. Zorzi, C. A. Perottoni, J. A. H. da Jornada, J. Mater. Sci. 37, 9 (2002) 1801-1807.

[7] ASTM C373-88 (Reapproved 2006), Standard Test Method for Water Absorption, Bulk Density, Apparent Porosity, and Apparent Specific Gravity of Fired Whiteware Products.

[8] A. P. Silva, A. M. Segadães, T. C. Devezas, Cerâmica 50, 316 (2004) 345-354.

[9] F. F. Lange, J. Am. Ceram. Soc. 72, 1 (1989) 3-15.

[10] R. A. Barbieri, J. E. Zorzi, C. A. Perottoni, Int. J. Appl. Ceram. Tech. (2012), Disponível em http://onlinelibrary. wiley.com/doi/10.1111/j.1744-7402.2011.00682.x/abstract [11] S. Novak, S. M. H. Olhero, J. M. F. Ferreira, A. Zupancic, Rheol. Acta 43 (2004) 559-566.

[12] B. C. Mutsuddy, R. G. Ford, Ceramic Injection Molding, Chapman Hall, UK (1995).

[13] B. Loebbecke, R. Knitter, J. Haußelt, J. Eur. Ceram. Soc. 29 (2009) 1595-1602.

[14] P. A. Ourique, Efeito das variáveis de processamento em misturas de alumina/ligantes orgânicos usados na moldagem por injeção em baixa pressão, Diss. Mestrado, PGMAT-UCS, RS (2012).

[15] L. Gorjan, A. Dakskobler, T. Kosmač, J. Am. Ceram. Soc. 95, 1 (2012) 188-193.

(Rec. 11/02/2012, Rev. 06/03/2012, Ac. 02/05/2012) 